\title{
Influence of Microcrystallinity on the CO/Pt(poly) Electrode Surface Using Sum Frequency Generation Microscopy Combined with Electrochemistry
}

Hao Li and Steven Baldelli*

Department of Chemistry, University of Houston, Houston, Texas 77204-5003, United States

\section{Supporting Information}

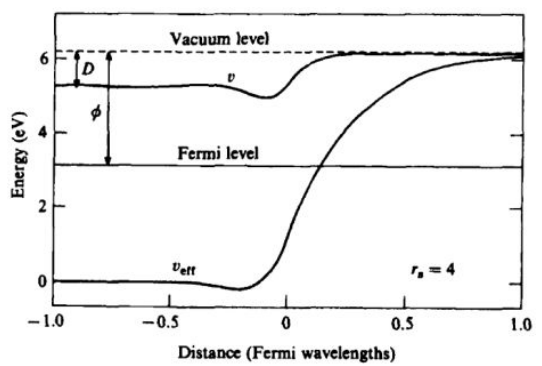

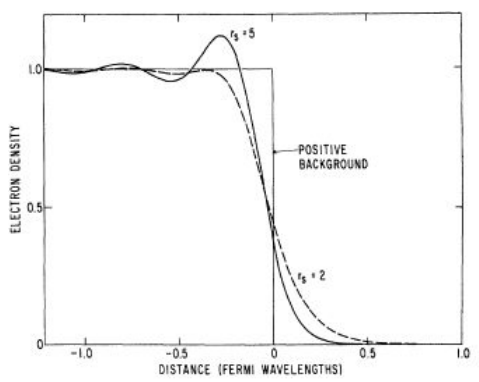

b

Figure $S 1$ (a) electrostatic potential, $v(z)$, a e-electron potential, $v_{\text {eff }}(z)$ near a jellium surface. (b) electron density profile for two choices of the background density, $r{ }^{1}{ }^{1}$

Self-designed SFGM-electrochem Cell
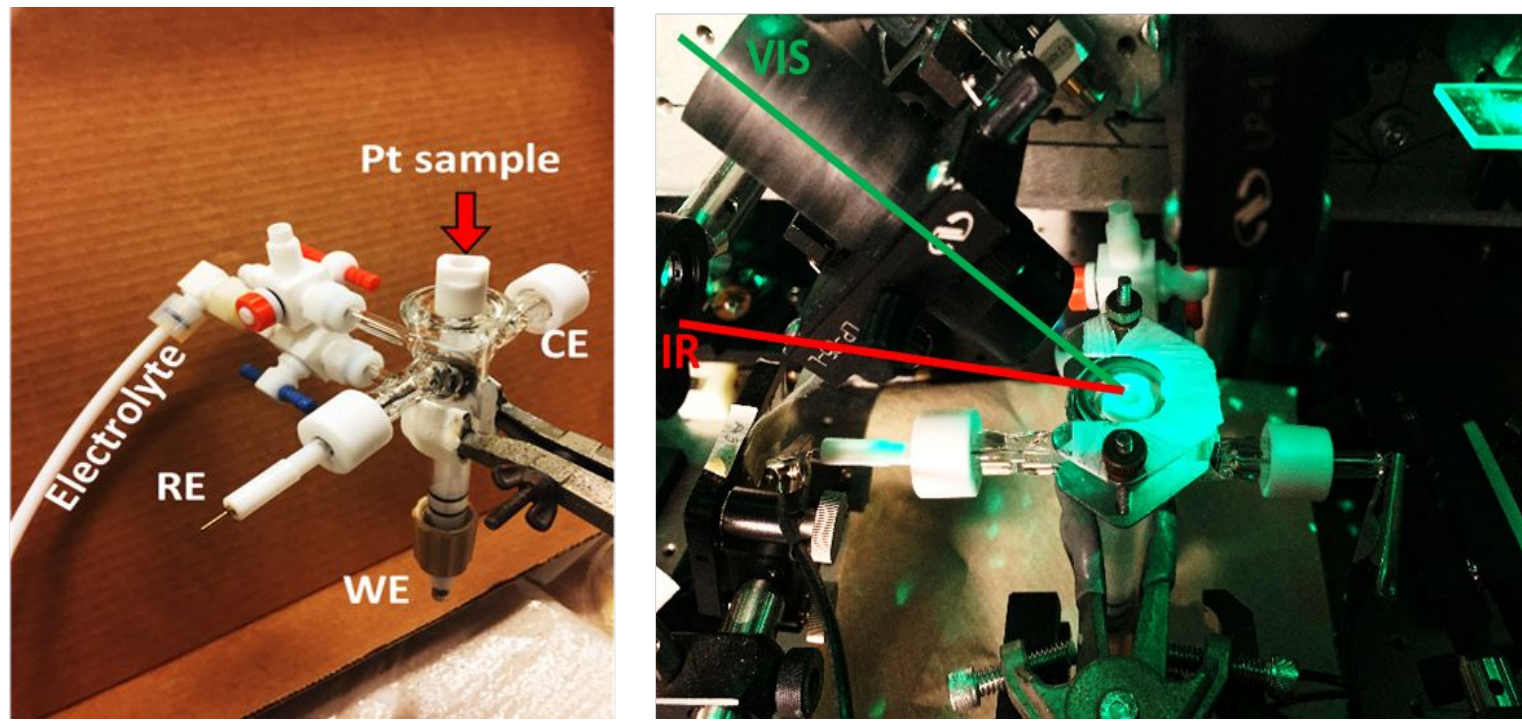

Figure S2 Left: Self-designed SFGM-electrochem cell. RE and CE were set on two sidearms. The Pt WE was assembled from top. Right: Schematic diagram of the cell assembled on SFG setup with incident beams.

The reference electrode (RE) and the counter electrode (CE) were fixed on the two sidearms. The polycrystalline Pt working electrode (WE) was stabilized on the center rotating rod by a homemade PTFE thread cap. The working electrode was connected from the bottom to the potentiostat with an aluminum rod inside the center rotating rod. After filling the electrolyte into the cell, $\mathrm{a} \mathrm{CaF}_{2}$ window was 
placed on the top of the cell and sealed by a Viton O-ring (McMASTER-CARR). The height of WE was adjusted by rotating the thread at the bottom of the cell.

\section{Spectra Normalization Process}

The spectra of the total imaging area and each domain were generated from Image software. In ImageJ, the specific area was selected by drawing the boundaries manually. The image stack loaded in the software contains the SFG images at different wavenumber channels. By defining the starting wavenumber and the wavenumber step between each image, a spectrum of the specific area was generated with the intensities at different wavenumbers. The output spectra were shown as the average intensity level of each pixel within each region. To show the signal contribution of each domain, the output spectra were multiplied with the area of each domain to obtain the area spectra. The measured areas of each domain through ImageJ are listed in Table 1. The dark pixels, representing little signal contribution, are not counted.

Table S1 Areas of each domain in SFG image

\begin{tabular}{|l|l|l|l|l|l|}
\hline Domain & Total & Pt (032) & Pt (111) & Pt (121) & Pt (122) \\
\hline Area (pixels) & 2873 & 1123 & 721 & 589 & 440 \\
\hline
\end{tabular}

Once the area spectra were generated, the peak intensity of the total area spectrum was set as 1 to obtain the normalization spectra as Figure 6 in the paper.

Nonlinear Fitting Process

The SFG spectrum of every single pixel was generated from SFG images stack in ImageJ software. The spectra were fitted with nonlinear fitting mode through Mathematica shown as equation 1:

$$
I_{S F}=\left|n e^{i p}+\frac{A_{1}}{-w+w_{1}-i f_{1}}+\frac{A_{2}}{-w+w_{2}-i f_{2}}+\frac{A_{3}}{-w+w_{3}-i f_{3}}\right|^{2}
$$

$I_{S F}$ represents the intensity of SF beam. $n$ is the amplitude of nonresonant susceptibility. p describes the phase difference between nonresonant and resonant susceptibility. $A$ is the amplitude of resonant susceptibility. $w$ and $f$ are the central wavenumber and bandwidth of different vibrational modes. Three resonant susceptibilities were fitted because of the three peak positions from the average spectrum of each domain. All of the parameters, $n, p, A, w$ and $f$ were saved in a $64 * 64$ matrix with the best fitting result. Each parameter matrix was mapped to correlate to the position of each pixel through Matlab, for example, the wavenumber map shown as Figure 8 in the paper.

Histograms of Stark Shift Pixels Fitting Map of Each Domain 


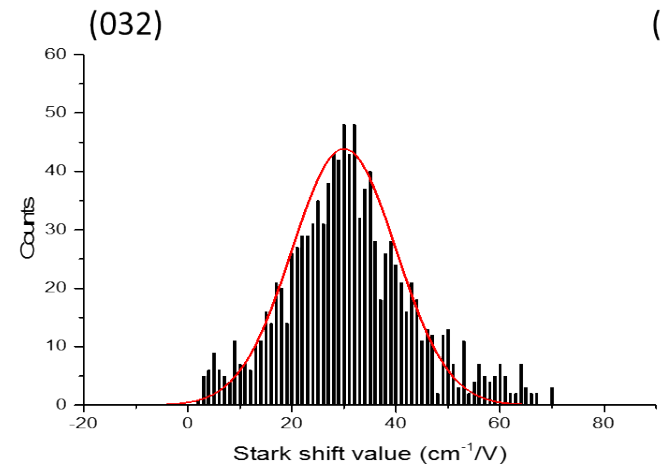

(111)
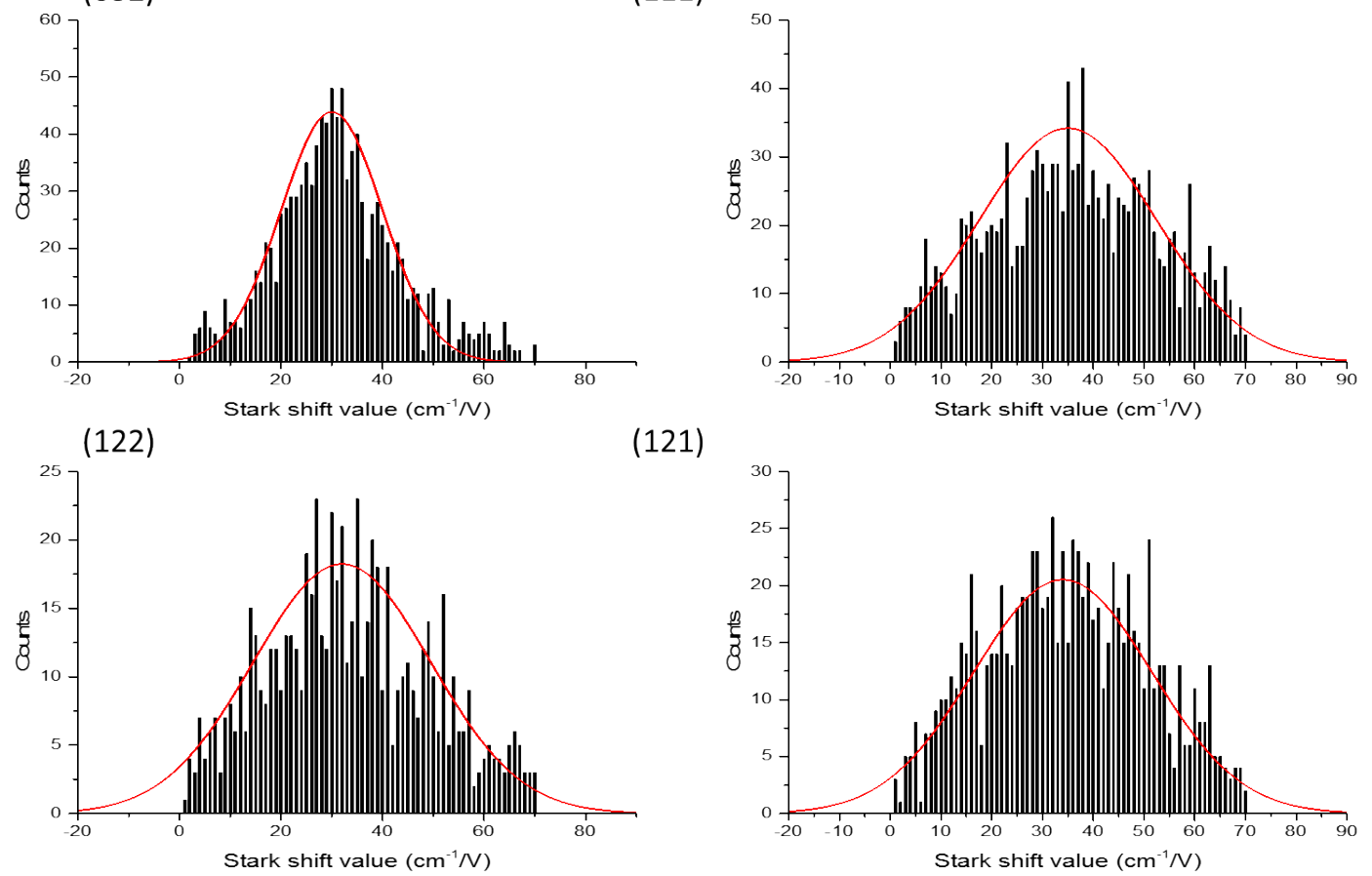

$(121)$

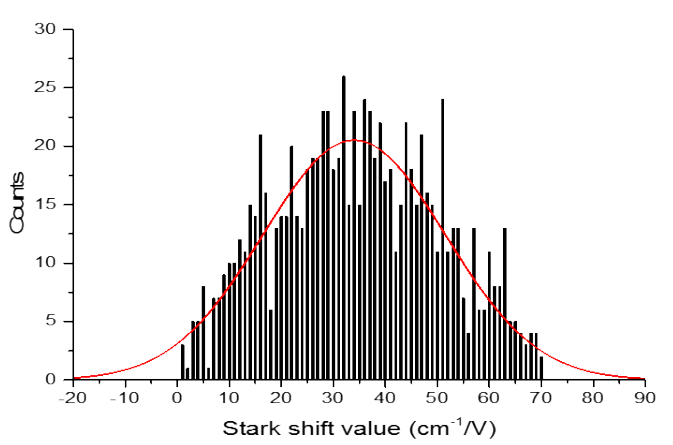

Figure S3 Histograms of Stark Shift Pixels Fitting Map of Each Domain

Table S2 CEO stretching mode wavenumber of different holding potentials vs. Ag/AgCl on different crystal facets with Stark shift values

\begin{tabular}{|c|c|c|c|c|c|c|}
\hline $\begin{array}{c}\text { Crystal } \\
\text { Facets }\end{array}$ & $\begin{array}{c}\text { Peak } \\
\text { Wavenumber } \\
\left(\mathrm{cm}^{-1}\right) \\
\text { at }-0.10 \mathrm{~V}\end{array}$ & $\begin{array}{c}\text { Peak } \\
\text { Wavenumber } \\
\left(\mathrm{cm}^{-1}\right) \\
\text { at } 0.10 \mathrm{~V}\end{array}$ & $\begin{array}{c}\text { Peak } \\
\text { Wavenumber } \\
\left(\mathrm{cm}^{-1}\right) \\
\text { at } 0.30 \mathrm{~V}\end{array}$ & $\begin{array}{c}\text { Peak } \\
\text { Wavenumber } \\
\left(\mathrm{cm}^{-1}\right) \\
\text { at } 0.50 \mathrm{~V}\end{array}$ & $\begin{array}{c}\text { Stark } \\
\text { shift } \\
\text { value } \\
\left(\mathrm{cm}^{-1} / \mathrm{V}\right)\end{array}$ & $\begin{array}{c}\text { Reference } \\
\text { value } \\
\left(\mathrm{cm}^{-1} / \mathrm{V}\right)\end{array}$ \\
\hline 032 & $2064 \pm 3$ & $2070 \pm 3$ & $2078 \pm 2$ & $2081 \pm 2$ & $30 \pm 3$ & $24\left(\mathrm{Pt} \mathrm{110)^{2 }}\right.$ \\
\hline 111 & $2050 \pm 4$ & $2054 \pm 3$ & $2064 \pm 4$ & $2072 \pm 4$ & $38 \pm 4$ & $30^{2} 36^{3-6}$ \\
\hline 121 & $2054 \pm 4$ & $2061 \pm 5$ & $2070 \pm 3$ & $2075 \pm 3$ & $36 \pm 3$ & $33(\mathrm{Pt} 335)^{7}$ \\
\hline 122 & $2068 \pm 3$ & $2073 \pm 2$ & $2078 \pm 3$ & $2085 \pm 2$ & $28 \pm 2$ & $33(\mathrm{Pt} \mathrm{332})^{8}$ \\
\hline Average & $2063 \pm 3$ & $2069 \pm 3$ & $2075 \pm 3$ & $2081 \pm 3$ & $30 \pm 3$ & $\begin{array}{c}28,{ }^{9} 30,10 \\
31,1135,12 \\
\text { (poly) }\end{array}$ \\
\hline
\end{tabular}

Work Function Adjustment and Fermi Level Shifting Schematic Diagrams 
A

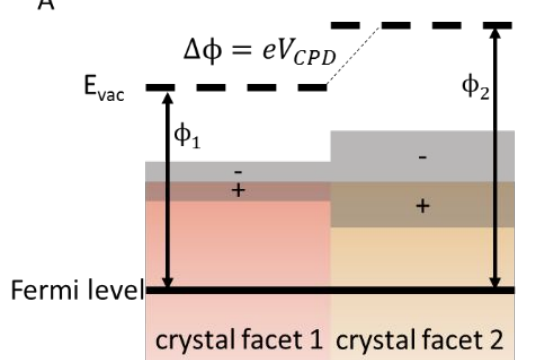

B

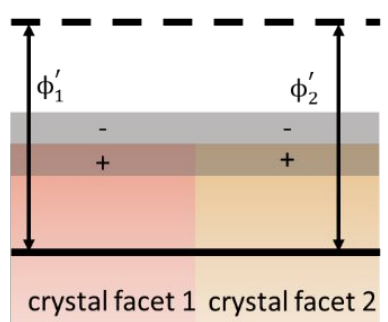

C

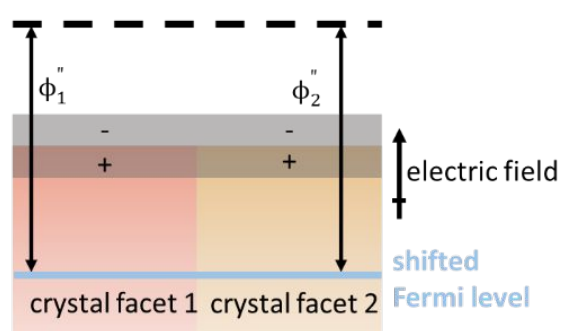

Figure S4 Leveling effect of work function (A) before and (B) after the contact between two crystal domains. The gray rectangle with charge represents the dipole formed at the electrode surface. (C) The shifting of Fermi levels with a positive applied potential.

\section{Structure Comparison between (121) and (335) Crystal Facets}

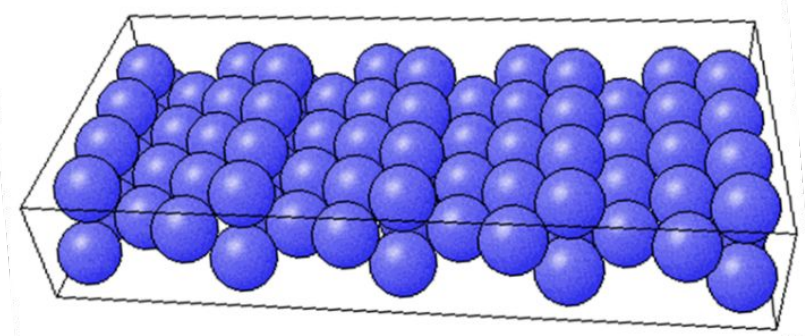

(335)

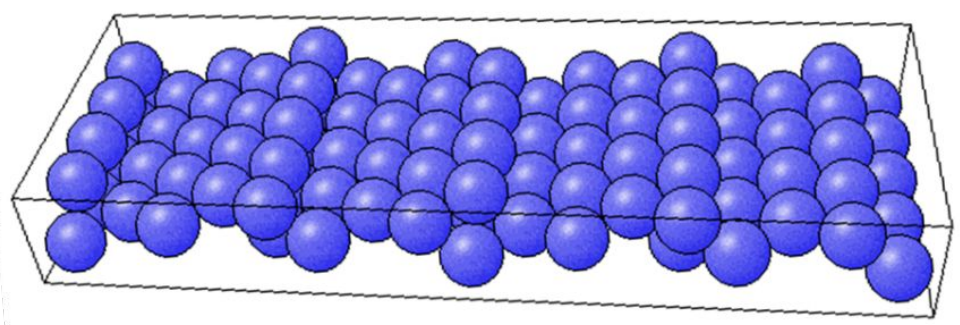

Figure S5 Structure comparison between (121) and (335) crystal facets generated from "Build and Analyze Lattices, Surfaces, And Clusters" (BALSAC) software ((C) K. Hermann, FHI 1991-2020)

\section{CO Coverage Calculation Process}




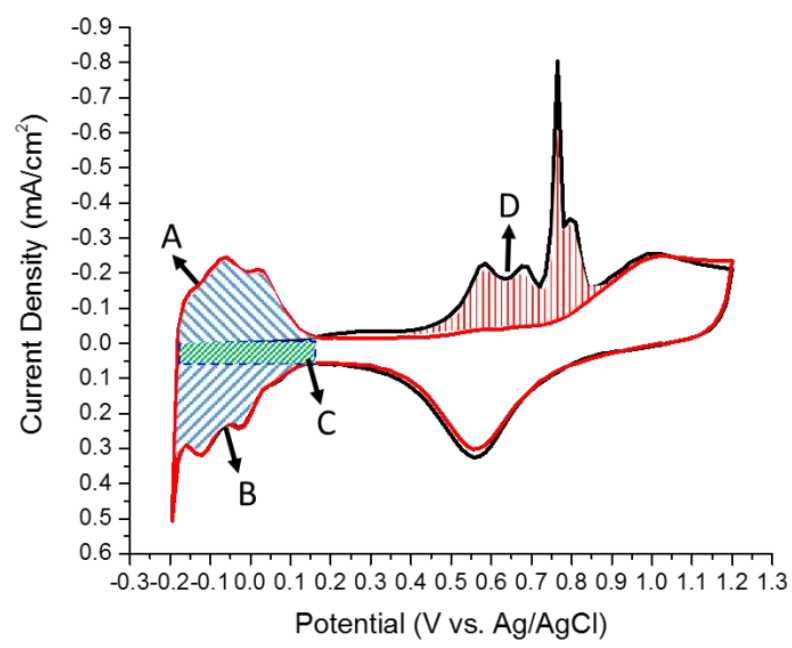

Figure S6 CV of CO on polycrystalline Pt in $0.1 \mathrm{H} 2 \mathrm{SO} 4$. Scan rate $=50 \mathrm{mV} / \mathrm{s}$. The black line and the red line represent the first and second scan, respectively. A: hydrogen desorption region; B: hydrogen adsorption region; C: double layer region within hydrogen $U P D$ region; $D$ : CO oxidation region.

The CO coverage was calculated as the charge ratio between the $\mathrm{CO}$ oxidation and the hydrogen desorption. The areas of the regions in Figure $\mathrm{S} 6$ were measured representing the charge. The hydrogen desorption region, labeled as region $\mathrm{A}$, was defined as the region between the first and second scan from $-0.20 \mathrm{~V}$ to $0.20 \mathrm{~V}$ vs. $\mathrm{Ag} / \mathrm{AgCl}$ during the anodic scan. The $\mathrm{CO}$ oxidation region was labeled as region D. The areas of region A and D were measured as 7168 (a.u.) and 9748 (a.u.), respectively. Because two electrons are transferred during the oxidation of one $\mathrm{CO}$ molecule, the ratio between the $\mathrm{CO}$ molecules and the hydrogen atoms is calculated as: $(9748 / 2) / 7168=0.68$.

A double layer is formed on the Pt electrode surface. While scanning the potential, the double layer is charged, resulting in a charging current. The double layer charging signal can be revealed from the flat line region from $0.2 \mathrm{~V}$ to $0.4 \mathrm{~V}$. Therefore, when calculate the area of hydrogen desorption and adsorption, the double layer charging current, shown as region $C$, should be subtracted by extending the flat line into the hydrogen UPD region. The area of hydrogen adsorption, shown as region $B$, was measured as 6963 (a.u.). The coverage of CO based on the hydrogen adsorption signal is calculated as: $(9748 / 2) / 6963=0.70$. 


\section{References}

(1) Zangwill, A. Physics at Surfaces; 1st ed.; Cambridge University Press: Great Britain, 1988.

(2) Tian, L.; Li, J.-T.; Ye, J.-Y.; Zhen, C.-H.; Sun, S.-G. In situ FTIR studies of coadsorption of CN- and CO on $\mathrm{Pt}(110)$ electrode surface. J. Electroanal. Chem. 2011, 662, 137-142.

(3) Lin, W. F.; Zei, M. S.; Eiswirth, M.; Ertl, G.; Iwasita, T.; Vielstich, W. Electrocatalytic Activity of RuModified Pt(111) Electrodes toward CO Oxidation. J. Phys. Chem. B 1999, 103, 6968-6977.

(4) Chang, S. C.; Weaver, M. J. Coverage - dependent dipole coupling for carbon monoxide adsorbed at ordered platinum(111) - aqueous interfaces: Structural and electrochemical implications. J. Chem. Phys. 1990, 92, 4582-4594.

(5) Stamenkovic, V.; Chou, K. C.; Somorjai, G. A.; Ross, P. N.; Markovic, N. M. Vibrational Properties of CO at the Pt(111)-Solution Interface: the Anomalous Stark-Tuning Slope. J. Phys. Chem. B 2005, 109, 678680.

(6) Kang, H.; Shin, S.; Park, Y.; Kang, H. Electric Field Effect on Condensed-Phase Molecular Systems. III. The Origin of the Field-Induced Change in the Vibrational Frequency of Adsorbed CO on Pt(111). J. Phys. Chem. C 2016, 120, 17579-17587.

(7) Kim, C. S.; Tornquist, W. J.; Korzeniewski, C. Infrared spectroscopy as a probe of carbon monoxide adsorption at platinum(335) under aqueous electrochemical conditions. J. Phys. Chem. 1993, 97, 64846491.

(8) Lebedeva, N. P.; Rodes, A.; Feliu, J. M.; Koper, M. T. M.; van Santen, R. A. Role of Crystalline Defects in Electrocatalysis: CO Adsorption and Oxidation on Stepped Platinum Electrodes As Studied by in situ Infrared Spectroscopy. J. Phys. Chem. B 2002, 106, 9863-9872.

(9) Lu, G. Q.; Lagutchev, A.; Dlott, D. D.; Wieckowski, A. Quantitative vibrational sum-frequency generation spectroscopy of thin layer electrochemistry: CO on a Pt electrode. Surf. Sci. 2005, 585, 3-16. (10) Lambert, D. K. Vibrational Stark effect of adsorbates at electrochemical interfaces. Electrochimica Acta 1996, 41, 623-630.

(11) Lu, G.-Q.; Sun, S.-G.; Chen, S.-P.; Cai, L.-R. Novel properties of dispersed Pt and Pd thin layers supported on GC for CO adsorption studied using in situ MS-FTIR reflection spectroscopy. J. Electroanal. Chem. 1997, 421, 19-23.

(12) lanniello, R.; Schmidt, V. M.; Stimming, U.; Stumper, J.; Wallau, A. CO adsorption and oxidation on Pt and Pt Ru alloys: dependence on substrate composition. Electrochim. Acta 1994, 39, 1863-1869. 